\title{
Prognostic Value of Dynamic Tissue Perfusion Measurements in Transplanted Kidneys
}

\author{
Thomas Scholbach $^{{ }^{*}}$, Hsin-Kai Wang ${ }^{2}$, Che-Chuan Loong ${ }^{3}$, Tsai-Hung $\mathrm{Wu}^{4}$ \\ ${ }^{1}$ Hospital for Children and Adolescents, Chemnitz Clinics, Chemnitz, Germany \\ ${ }^{2}$ Department of Radiology, Taipei Veterans General Hospital and School of Medicine, \\ National Yang-Ming University, Taipei, Taiwan \\ ${ }^{3}$ Department of Surgery, Taipei Veterans General Hospital and School of Medicine, \\ National Yang-Ming University, Taipei, Taiwan \\ ${ }^{4}$ Department of Internal Medicine, Taipei Veterans General Hospital and School of Medicine, \\ National Yang-Ming University, Taipei, Taiwan \\ Email: ${ }^{*}$ t.scholbach@skc.de, ushkwang@gmail.com, ccloong@vghtpe.gov.tw, thwu@vghtpe.gov.tw
}

Received 12 December 2013; revised 12 January 2014; accepted 20 January 2014

Copyright (c) 2014 Thomas Scholbach et al. This is an open access article distributed under the Creative Commons Attribution License, which permits unrestricted use, distribution, and reproduction in any medium, provided the original work is properly cited. In accordance of the Creative Commons Attribution License all Copyrights @ 2014 are reserved for SCIRP and the owner of the intellectual property Thomas Scholbach et al. All Copyright (C) 2014 are guarded by law and by SCIRP as a guardian.

\section{ABSTRACT}

Background: The dynamic tissue perfusion measurement in renal transplants has demonstrated its potential to correlate microvascular perfusion changes with histologic changes in the renal cortex [1]. Cross sectional studies have shown that the cortical perfusion depends on the time elapsed after transplantation [2,3] but has not yet been applied as a prognostic marker. Therefore, we compared in a prospective 6-year study the initial perfusion measurements with the outcome of the graft function. Methods: In 78 renal graft recipients, standardized color Doppler sonographic videos were recorded and cortical perfusion was measured in well-defined regions of interest with the PixelFlux-software. Results: At the beginning of the study, prospectively failing grafts (requiring dialysis) had a significantly lower cortical perfusion compared to grafts with preserved function (0.40 vs. 0.57 $\mathrm{cm} / \mathrm{s}$ average perfusion intensity of the entire cortex). The interval between perfusion measurement and graft failure was 2.17 years ( 0 - 6 years). Conclusions: Thus, dynamic tissue perfusion measurement may have a role in the prospective evaluation of renal transplant function.

\section{KEYWORDS}

\section{Color Doppler Ultrasound; Microvasculature; Perfusion Measurement; Prognostic Value; Renal Transplant}

\section{Introduction}

Biopsy of renal transplant remains the cornerstones of renal transplant evaluation, which provides guidance for treatment of graft dysfunction. However, it carries risks of procedure-related complications from pain to graft loss. Repeated renal biopsy is restricted to patients with deteriorating renal function or patients who receive protocol biopsies. Dynamic Tissue Perfusion Measurement (DTPM) of the renal transplant's cortex is a non-invasive method to describe renal transplants' cortical perfusion and has demonstrated its performance in normal kidneys as well as renal transplants. Previous studies demon-

\footnotetext{
${ }^{*}$ Corresponding author.
}

strated the correlation between cortical perfusion and histological criteria based on the Banff-classification.

\section{Objectives}

In this study, we compared the initial cortical dynamic perfusion measurement with the functional graft outcome after a six-year follow up.

\section{Methods}

\subsection{Patients}

This study was approved by the Institutional Review Board. Seventy-eight patients received DTPM study be- 
tween December 2005 and September 2008. 41 males and 37 females were included in this study; mean age when receiving renal transplantation was $41.0 \pm 12.6$ years (range 6 - 72 years); 68 received renal transplantation from deceased donor, 6 received simultaneous pancreas and kidney transplantation, 4 received living-related renal transplantation. Mean time between the patient receiving renal transplantation and DTPM was 44.8 months.

\subsection{Color Doppler Sonography}

Color Doppler sonography was performed by a single investigator (H.-K. W.). An ATL HDI-5000 ultrasound unit (Philips Medical System, Bothell, WA, USA) equipped with a linear L12-5 MHz transducer was used in this study. A standardized preset of the color Doppler imaging parameters was applied for imaging acquisition in all patients: imaging depth, $4 \mathrm{~cm}$; color Doppler frequency, $6 \mathrm{MHz}$; pulse repetition frequency, $1000 \mathrm{~Hz}$; color gain, 79\%; wall filter: medium; flow option: medium velocity; color map: 3 . A longitudinal section of the proximal outer cortex of the transplant kidney was selected for scanning. The imaging field remained unchanged during scanning. The pressure of the ultrasound transducer to the skin was kept minimal. Two color Doppler cine loops video encompassing at least two full heart cycles (40 frames with a frame rate of 9 - 10/sec) were recorded and stored in a Digital Imaging and Communications in Medicine (DICOM) format.

\subsection{Dynamic Tissue Perfusion Measurement}

Dynamic tissue perfusion measurements (DTPM) of recorded color Doppler videos were performed by a single investigator (T.S.) blinded to the clinical outcome using the software Pixel Flux [4]. The central segment of the outer cortex was selected for dynamic tissue perfusion measurement with the following steps:

The video was opened, then the color bar was read out and all hues, maximum encoded velocity and distances were calibrated automatically. The region of interest (ROI), consisting of a parallelogram, was selected (see Figure 1). The four corners of the parallelogram were set as follows: The first one at the center of the outer edge of a medullary pyramid, the second one at the center of the outer edge of the neighboring medullary pyramid, the third and fourth ones at the renal surface perpendicular to the first two corners. The ROI was divided into 10 consecutive slices each encompassing $10 \%$ of the entire cortical thickness.

Each slice stretched from the left to the right border of the ROI and had a height which encompassed $10 \%$ of the ROI's entire height. The $10 \%$ slices were labeled 10 to

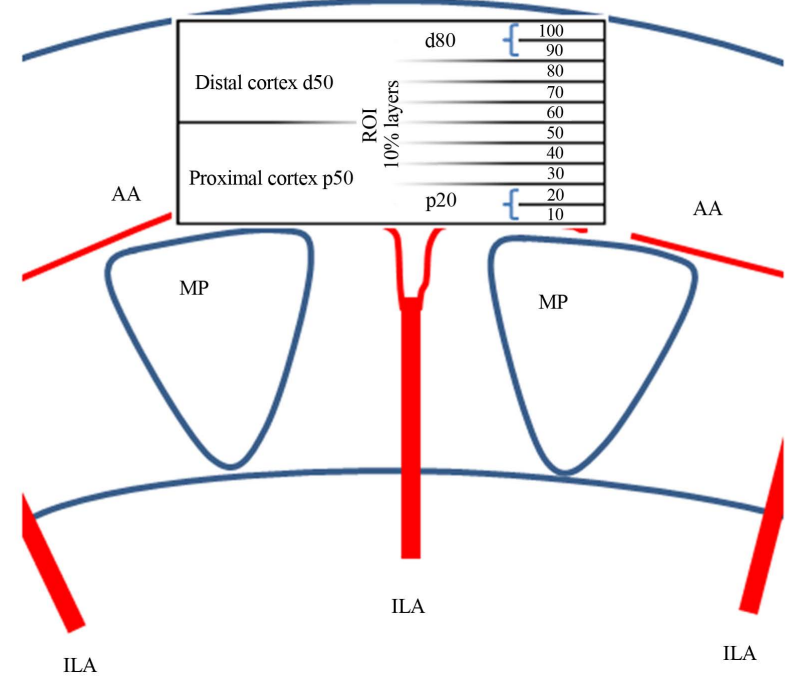

Figure 1. Definition of the cortical region of interest (ROI) for dynamic tissue perfusion measurements.

100 from proximal to the subcapsular cortical layer (Figure 1). The mean velocity value of all colored pixels inside the ROI and the area of all colored pixels were calculated by the software, identifying the beginning and end of a complete heart cycle within the video automatically and restricting all calculations to a full heart cycle.

\subsection{Monitoring of Patients' Outcome}

The graft function and clinical situation of the patients were monitored periodically after DTPM study for 6 years. Initiation of hemodialysis due to a deteriorated graft function was regarded as graft failure.

\subsection{Statistics}

Data were statistically analysed using the SPSS 21 software (IBM). Groups were compared applying the MannWhitney-U-test. P-values less than 0.05 were considered to be statistically significant.

\section{Results}

Twenty-nine patients developed graft failure and 13 patients died during the 6-year follow-up. The ages of the subgroups at transplantation, Doppler investigation and death are given in Table 1(a). No significant differences of age at transplantation existed between prospectively failing and non-failing grafts (Table 1(b)). This means that the age had no relevant influence on cortical perfusion of the transplants. Therefore, age alone is not responsible for the perfusion loss in prospectively failing grafts. The cortical perfusion of the entire renal cortex was significantly lower in those grafts, which developed graft failure later on $(\mathrm{p}=0.030)$ (Figure 2). The perfu- 
Table 1. (a) Ages of transplanted patients at transplantation, Doppler examination, graft failure and death; (b) No significant age differences exist between patients with failing and non-failing grafts.

(a)

\begin{tabular}{ccccccc}
\hline & Age RTX [a] & Age Doppler [a] & Age death [a] & $\begin{array}{c}\text { Interval } \\
\text { RTX-Doppler [a] }\end{array}$ & $\begin{array}{c}\text { Interval } \\
\text { RTX-Failure [a] }\end{array}$ & $\begin{array}{c}\text { Interval } \\
\text { Doppler-Failure [a] }\end{array}$ \\
\hline Mean & 40.97 & 44.82 & 51.15 & 3.86 & 7.25 & 2.17 \\
Maximum & 72.00 & 75.00 & 60.00 & 20.00 & 20.00 & 6.00 \\
Minimum & 6.00 & 13.00 & 30.00 & 0.00 & 1.00 & 0.00 \\
Standard deviation & 12.79 & 12.55 & 7.63 & 4.85 & 5.59 & 1.82 \\
Number & 78 & 78 & 13 & 78 & 23 & 22 \\
\hline
\end{tabular}

(b)

\begin{tabular}{cccc}
\hline Mann-Whitney-U-test $\mathrm{p}=0.930$ & Age & N & SD \\
\hline No graft failure & 45.26 & 49 & 13.08 \\
graft failure & 44.09 & 29 & 12.03 \\
\hline
\end{tabular}

sion differences between failing and non-failing grafts gradually increased from central to peripheral layers of the cortex (Figure 3). The Pixel Flux technique is sensible enough to detect relevant perfusion differences even among ten very thin consecutive cortical layers.

\section{Discussion}

The outcome of a renal transplant basically depends on the state of its microvasculature. Tissue integrity and renal function need, beside other important prerequisites, a sufficient supply of oxygen and other substances transported by blood [5].

The tubulointerstitium, making up approximately 80\% of the renal volume, has a high energy demand and is susceptible to injury in situations of relative nutrient deprivation [6]. Ischemia and reperfusion trigger momentary and long-lasting remodeling processes as actin-disarrangement in renal cortical arterioles [7]. Ischemia was followed by persistent vasoconstriction in a rat model and led to lasting oxidant stress after recovery from acute kidney injury which fuelled fibrosis [8]. This may contribute to an ever accelerating deterioration after perfusion falls short of a certain threshold. Within this context it is interesting that DTPM could demonstrate a significant correlation of the renal transplant fibrosis degree and tissue perfusion [9]. The potential of this technique is further emphasized by data which show that in patients with stable chronic renal failure, the distal cortical perfusion correlates to cystatin levels [10]. In renal transplants, the cortical DTPM, but not the widely used intrarenal Doppler Resistance Index (RI), correlated significantly ith the estimated glomerular filtration rate (eGFR) and donor-derived factors. Cortical PI values were neither correlated with RI of segmental arteries, nor with pulse

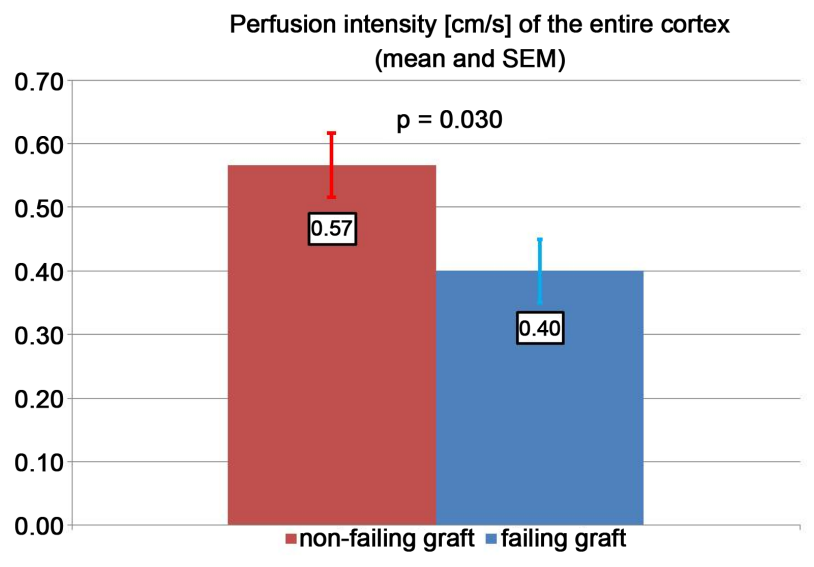

Figure 2. Cortical perfusion intensity in failing and nonfailing grafts.

w pressure, serum creatinine or the recipient's age-indicating that the cortical DTPM is a distinct intrarenal hemodynamic parameter [11].

RI values of segmental arteries in a renal transplant recipient depend mainly on recipient-related factors affecting vascular compliance, such as pulse pressure or recipient age. Whereas DTPM characterize intrarenal factors, in transplanted as well as in native kidneys [10,11]. RI also demonstrated less sensitivity compared to DTPM in transplants with increasing peritubular inflammation [1].

Stagnation of blood flow in peritubular capillaries induced chronic hypoxia at an early stage in a rat glomerulonephritis model, which was followed by progressive tubulointerstitial injury and a loss of peritubular capillaries [12]. Renal hypoxia itself can intensify or stimulate the invasion of inflammatory cells [5] and thus can aggravate inflammation. Moreover, hypoxia can stimulate fibrosis [13]. Therefore, notwithstanding the undisputed 


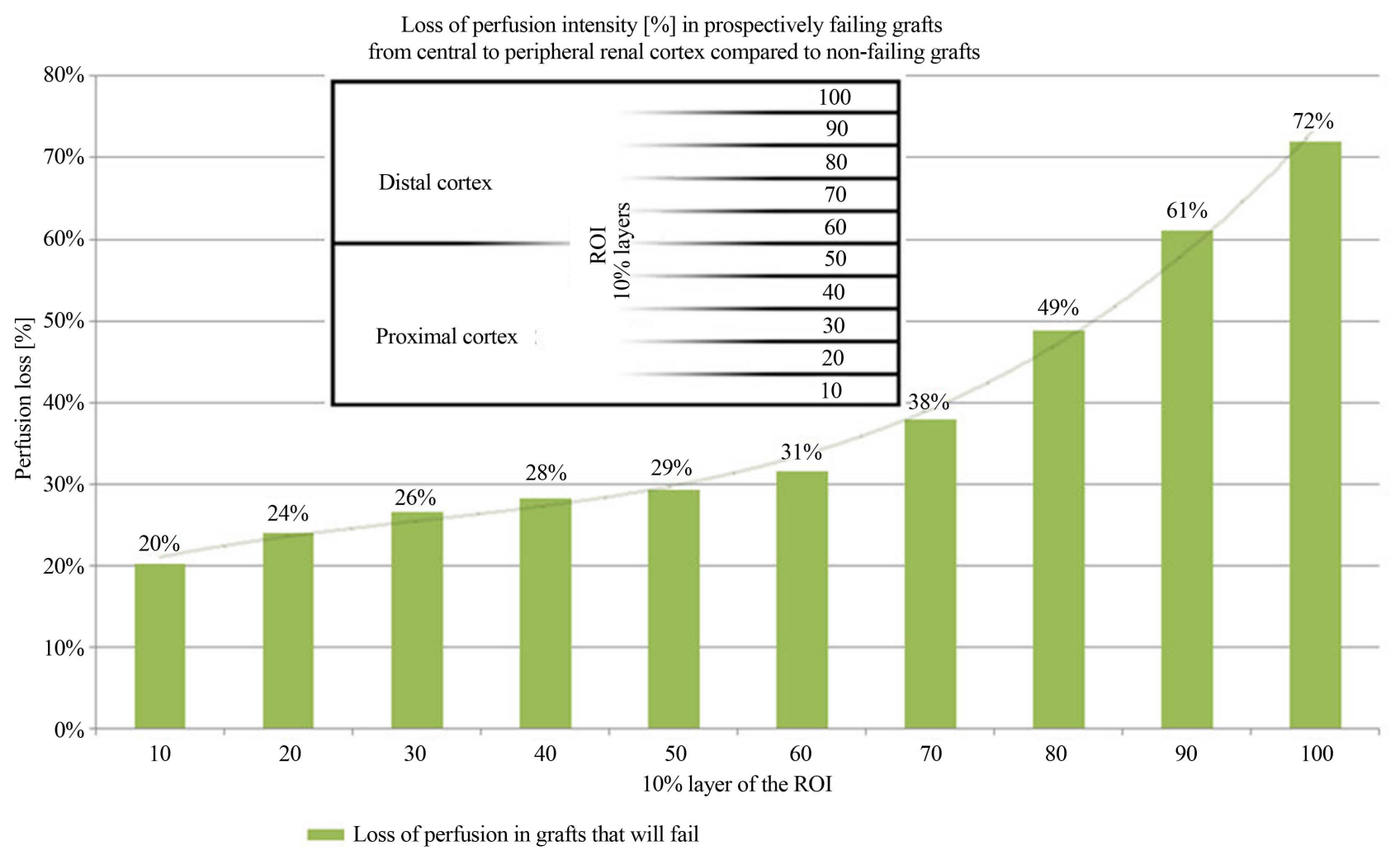

Figure 3. Perfusion intensity differences in $\mathbf{1 0}$ consecutive horizontal cortical layers in failing and non-failing grafts.

immunological mechanisms driving the transplant into insufficiency, it is worthwhile to have a close look onto the actual perfusion of the renal microvasculature. A diminution of its perfusion mirrors the functional state of the transplant, no matter if the perfusion decrease is the cause or if the functional deterioration is the consequence of the perfusion decline. Renal microvasculature is hierarchically structured. Rather wide interlobar arteries enter the renal cortex branching into arcuate arteries which give rise to the even narrower interlobular arteries. On their course to the outer regions of the cortex these tiny vessels distribute blood via further branches to the neighboring glomeruli. This way each layer is perfused by less blood than its preceding layer below. This natural gradient $[2,3,14]$ is enlarged by any chronic process that is either affecting the arterial walls or the glomerular transit. A certain thickening of the arterial walls will reduce the blood flow stronger in small sized vessels than in wider vessels. The DTPM is in accordance with this theoretical background. It demonstrates a loss of perfusion in prospectively failing grafts in general, but within the most distal cortical layers the reduction of perfusion is most pronounced (Figure 3). These are the layers where the tiniest vessels prevail, which are obliterated first, already in the beginning of an insidious chronic disease. Remarkably, these Doppler examinations preceded the graft failure by up to 6 years (Table 1(a)).
Similar early effects on the renal microvasculature could be demonstrated in a previous study in renal transplants [1] and diabetic kidneys before the onset of microalbuminuria [own paper accepted for publication].

The mean interval between DTPM and renal transplant failure was 2.17 years $(0-6$ years) (Table $1(\mathrm{a}))$. Thus, PixelFlux provides a novel approach to measure microvascular damage of renal transplants years before the functional impact is threatening the survival of the organ.

Aschwanden et al. [15] found in a series of 80 transplantations that an immediate postoperative determination of the RI is not predictive of early transplant function, but that the intrarenal resistance rises within a few days from normal to pathological levels when delayed graft functionis present. This means, the RI falls short as a prognostic parameter but merely describes general vascular conditions in the recipient or comments on already evident clinical and laboratory findings. DTPM might offer an alternative since it reflects the state of the microvasculature in up to 10 subsequent cortical layers better than the simple perfusion velocity ratio within few interlobar arteries, as offered by RI. RI measurements basically cannot give a clue to tissue perfusion since they do not refer to true flow velocities and the perfused area inside the tissue.

\section{Conclusion}

Our study demonstrated that the perfusion of the renal 
transplant is associated with functionally significant changes of the microvessels within the cortex of the graft. DTPM showed that a significant reduction of predominantly peripheral cortical perfusion is associated with later graft failure. This reduction of the microvascular perfusion preceded the function loss of the organs by up to 6 years.

\section{Transparency Declarations}

Thomas Scholbach was involved in the development of the Pixel Flux-software and holds shares in Chameleonsoftware. The other authors have no conflict of interest.

\section{REFERENCES}

[1] T. Scholbach, H. K. Wang, A. H. Yang, C. C. Loong and T. H. Wu, "Correlation of Histopathologic and Dynamic Tissue Perfusion Measurement Findings in Transplanted Kidneys,” BMC Nephrology, Vol. 14, 2013, p. 143. http://dx.doi.org/10.1186/1471-2369-14-143

[2] T. Scholbach, E. Girelli and J. Scholbach, "Dynamic Tissue Perfusion Measurement: A Novel Tool in Follow-Up of Renal Transplants,” Transplantation, Vol. 79, No. 12, 2005, pp. 1711-1716. http://dx.doi.org/10.1097/01.TP.0000164145.89275.02

[3] T. Scholbach, E. Girelli and J. Scholbach, "Tissue Pulsatility Index: A New Parameter to Evaluate Renal Transplant Perfusion,” Transplantation, Vol. 81, No. 5, 2006, pp. 751-755.

http://dx.doi.org/10.1097/01.tp.0000201928.04266.d2

[4] Chameleon-Software: PixelFlux, 2009. www.chameleon-software.de

[5] F. Palm and L. Nordquist, "Renal TubulointerstitialHypoxia: Cause and Consequence of Kidney Dysfunction," Clinical and Experimental Pharmacology \& Physiology, Vol. 38, No. 7, 2011, pp. 474-480. http://dx.doi.org/10.1111/j.1440-1681.2011.05532.x

[6] K. S. Hodgkins and H. W. Schnaper, "TubulointerstitiaIInjury and the Progression of Chronic Kidney Disease," Pediatric Nephrology, Vol. 27, No. 6, 2012, pp. 901-909.

[7] O. Kwon, C. L. Phillips and B. A. Molitoris, "Ischemia Induces Alterations in Actin Filaments in Renal Vascular Smooth Muscle Cells," American Journal of Physiology Renal Physiology, Vol. 282, No. 6, 2002, pp. F1012F1019.

[8] D. P. Basile, E. C. Leonard, A. G. Beal, D. Schleuter and J. Friedrich, "Persistent Oxidative Stress Following Renal
Ischemia-Reperfusion Injury Increases ANG II Hemodynamicand Fibrotic Activity," American Journal of Physiology Renal Physiology, Vol. 302, No. 11, 2012, pp. F1494-F1502.

http://dx.doi.org/10.1152/ajprenal.00691.2011

[9] T. Syversveen, K. Brabrand, K. Midtvedt, E. H. Strom, A. Hartmann and A. E. Berstad, "Non-Invasive Assessment of Renal Allograft Fibrosis by Dynamic Sonographic Tissue Perfusion Measurement,” ActaRadiologica, Vol. 52, No. 8, 2011, pp. 920-926. http://dx.doi.org/10.1258/ar.2011.110215

[10] A. Lubas, R. Ryczek, G. Kade, J. Smoszna and S. Niemczyk, "Impact of Cardiovascular Organ Damage on Cortical Renal Perfusion in Patients with Chronic Renal Failure,” BioMedResearch International, 2013, Article ID: 137868.

[11] E. Marti, I. P. Bergmann, D. E. Uehlinger, F. J. Frey and U. Eisenberger, "Donor Effect on Cortical Perfusion Intensity in Renal Allograft Recipients: A Paired Kidney Analysis," American Journal of Nephrology, Vol. 33, No. 6, 2011, pp. 530-536. http://dx.doi.org/10.1159/000328383

[12] M. Matsumoto, T. Tanaka, T. Yamamoto, E. Noiri, T. Miyata, R. Inagi, T. Fujita and M. Nangaku, "Hypoperfusion of Peritubular Capillaries Induces Chronic Hypoxia before Progression of Tubulointerstitial Injury in a Progressive Model of Rat Glomerulonephritis," Journal of the American Society of Nephrology: JASN, Vol. 15, No. 6, 2004, pp. 1574-1581. http://dx.doi.org/10.1097/01.ASN.0000128047.13396.48

[13] Z. Wang, L. Tang, Q. Zhu, F. Yi, F. Zhang, P. L. Li and N. Li, "Hypoxia-Inducible Factor-1Alpha Contributes to the Profibrotic Action of Angiotensin II in Renal Medullary Interstitial Cells,” Kidney international, Vol. 79, No. 3, 2011, pp. 300-310. http://dx.doi.org/10.1038/ki.2010.326

[14] T. Scholbach, I. Dimos and J. Scholbach, “A New Methodof Color Doppler Perfusion Measurement via Dynamic Sonographic Signal Quantification in Renal Parenchyma,” Nephron Physiology, Vol. 96, No. 4, 2004, pp. 99-104.http://dx.doi.org/10.1159/000077380

[15] M. Aschwanden, M. Mayr, S. Imfeld, J. Steiger, K. A. Jaeger and C. Thalhammer, "Rapid Adaptation of the Intrarenal Resistance Index after Living Donor Kidney Transplantation,” Nephrology, Dialysis, Transplantation: Official Publication of the European Dialysis and Transplant Association-European Renal Association, Vol. 24, No. 4, 2009, pp. 1331-1334. 